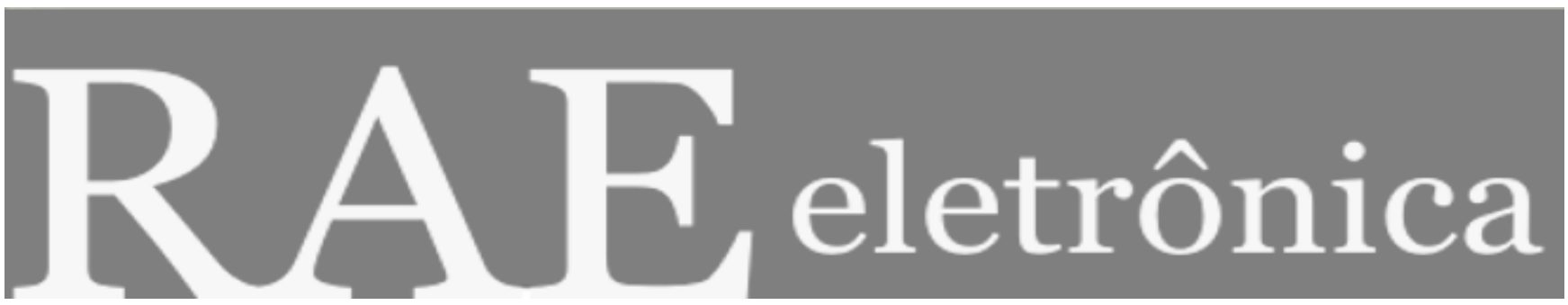

\title{
ADMINISTRAÇÃO DE SEGUROS NO TRANSPORTE DE CARGAS
}

Por

\section{Augusto Hauber Gameiro}

José Vicente Caixeta Filho

RAE-eletrônica, Volume 1, Número 1, jan-jun/2002.

http://www.rae.com.br/eletronica/index.cfm?FuseAction=Artigo \&ID=399\&Secao=OPERA/LOGI\&Volume=1\&Numero=1 $\& A n o=2002$

CCopyright, 2002, RAE-eletrônica. Todos os direitos, inclusive de tradução, são reservados. É permitido citar parte de artigos sem autorização prévia desde que seja identificada a fonte. A reprodução total de artigos é proibida. Os artigos só devem ser usados para uso pessoal e nãocomercial. Em caso de dúvidas, consulte a redação: redacao@,rae.com.br.

A RAE-eletrônica é a revista on-line da FGV-EAESP, totalmente aberta e criada com o objetivo de agilizar a veiculação de trabalhos inéditos. Lançada em janeiro de 2002, com perfil acadêmico, é dedicada a professores, pesquisadores e estudantes. Para mais informações consulte o site www.rae.com.br/eletronica.

RAE-eletrônica

ISSN 1676-5648

(C2002 Editora: Fundação Getulio Vargas - Escola de Administração de Empresas de São Paulo.

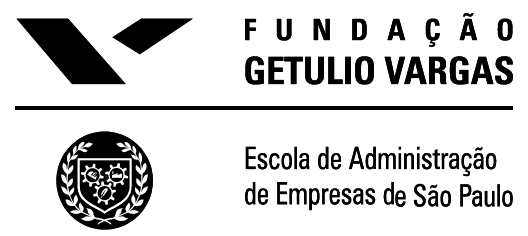




\title{
ADMINISTRAÇÃO DE SEGUROS NO TRANSPORTE DE CARGAS
}

\section{Augusto Hauber Gameiro}

Pesquisador do Centro de Estudos Avançados em Economia Aplicada (CEPEA/ESALQ/USP).

Engenheiro Agrônomo pela Universidade Federal de Pelotas, Mestre em Economia Aplicada pela ESALQ/USP e Doutorando em Economia Aplicada pela mesma Escola.

E-mail: ahgameir@terra.com.br

Endereço: ESALQ/USP, Av. Pádua Dias, 11, Piracicaba SP, 13400-970

Interesses de pesquisa: Gestão Logística.

\section{José Vicente Caixeta Filho}

Professor Associado do Departamento de Economia, Administração e Sociologia da ESALQ/USP.

Engenheiro Civil pela Escola Politécnica da USP, Master in Economics pela University of New

England (Austrália), Doutor em Engenharia de Transportes pela Escola Politécnica da USP.

E-mail: jvcaixet@,esalq.usp.br

Endereço: ESALQ/USP, Av. Pádua Dias, 11, Piracicaba SP, 13400-970

Interesses de pesquisa: Gestão Logística.

\section{RESUMO}

Este trabalho analisa a administração de seguros de carga por parte de empresas de transporte rodoviário, com ênfase no tratamento do risco de roubo de cargas. Esses seguros apresentam peculiaridades que os torna, muitas vezes, instrumentos complexos. Sendo assim, as empresas acabam encontrando dificuldade na sua operacionalização, principalmente no atual contexto, no qual o evento roubo apresenta-se como um forte empecilho ao desempenho do setor. Há uma evidente necessidade de revisão da gerência de risco por parte dessas empresas. A heterogeneidade do setor exige novos produtos de seguro mais adequados às diferentes situações. Faz-se necessário, ainda, uma profunda revisão da relação entre transportadores e embarcadores.

\begin{abstract}
This paper analyses the management of cargo insurance by trucking companies, with emphasis on the treatment of cargo theft. This type of insurance has peculiarities that make it, most of the time, a very complex tool to be used. Consequently, companies face difficulties to manage them, mainly at the present context, where the theft is a bottleneck for the performance of the transportation sector. There is a clear need of revising risk management strategies by the firms. The sector's particularities require new insurance products, more adaptable to different situations. It's also necessary a detailed revision of the relations between carriers and shippers.
\end{abstract}

\section{PALAVRAS-CHAVES}

Seguros, transporte, risco, roubo, carga.

\section{KEY WORDS}

Insurance, transport, risk, theft, cargo. 


\section{INTRODUÇÃO}

A ocorrência frequente de desaparecimento de cargas vem afetando consideravelmente o desempenho do setor de Transporte Rodoviário de Carga ${ }^{1}$ (TRC) no Brasil e no mundo (Mooney, 1994). Para se ter uma idéia, no ano de 1998, foram registrados no Estado de São Paulo 1.334 eventos, totalizando US\$ 122,46 milhões em cargas roubadas ${ }^{2}$.

Métodos tradicionais de Gerência de Risco na atividade, como a utilização de seguro para carga (Mehr et al., 1963), estão sendo questionados em relação à sua eficiência e disponibilidade.

Acredita-se que a natureza desses seguros - muitas vezes firmados através de contratos complexos - possa ser um ponto de estrangulamento na gerência de risco dessas empresas. Isto é, pode haver uma certa falta de conhecimento dos administradores em relação a esse assunto, principalmente no atual contexto onde o risco de ocorrência de roubo é uma ameaça clara ao setor.

Em vista disso, o objetivo deste trabalho é o de analisar os aspectos relacionados à administração da carteira de seguros de carga nas empresas de transporte. O texto está estruturado da seguinte forma: inicialmente são expostas as principais características do roubo de cargas no País, bem como dos seguros de cargas disponíveis no mercado. Em seguida, é abordada a administração dos seguros, propriamente dita, por parte das empresas pesquisadas. Ao final, são apresentadas as conclusões do trabalho.

\section{ROUBO DE CARGA E SEGURO}

Para a análise em questão, é considerado "roubo de carga"”3 a situação em que indivíduos tomam posse ilegalmente de parte ou da totalidade da carga de um veículo transportador, podendo ocorrer, no último caso, o desaparecimento ou não do veículo concomitantemente com a mercadoria roubada, caso em que se utiliza a denominação "desvio de carga".

A título de ilustração, os valores envolvidos nos eventos ocorridos nos Estados de São Paulo e Rio de Janeiro constam na Figura 1.

Figura 1. Evolução anual dos valores de roubos de cargas nos Estados do Rio de Janeiro e São Paulo

(Fonte: Assessorias de Segurança do SINDICAR

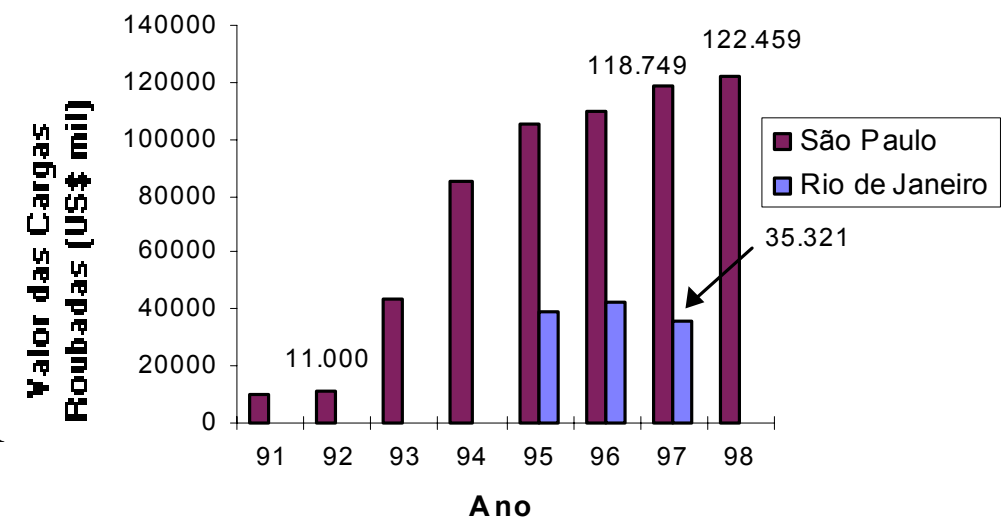
GA e SETCESP).

\footnotetext{
${ }^{1}$ Para maiores informações sobre a estrutura de mercado do Transporte Rodoviário de Cargas, consultar Castro, 1988 e Uelze, 1977.

${ }^{2}$ Assessoria de Segurança do Sindicato das Empresas de Transporte de Carga de São Paulo e Região (SETCESP).

${ }^{3} \mathrm{O}$ termo "roubo de carga" será genericamente utilizado para mencionar os casos de roubo propriamente dito, furto ou desvio de carga.
} 
Observa-se que as perdas com cargas roubadas chegaram a US\$ 122 milhões no Estado de São Paulo no ano de 1998. Os Estados de São Paulo e Rio de Janeiro são as duas regiões onde o problema apresenta-se mais evidente no País.

Os dados de ocorrências também indicam a preferência pelo roubo de mercadorias com elevado valor agregado e de fácil distribuição no mercado, tais como: cigarros, medicamentos, aparelhos eletrônicos, etc., ou seja, as denominadas "cargas secas".

Em relação aos seguros de carga disponíveis no mercado brasileiro, menciona-se três: o denominado "RR" (Riscos Rodoviários), que é um seguro com ampla cobertura e obrigatório aos embarcadores (proprietários das mercadorias); o RCTR-C, seguro de responsabilidade civil obrigatório para transportadores que assegura-os em casos de perdas ocorridas na carga que estava sob sua responsabilidade; e o RCF-DC, também um seguro de responsabilidade de transportadores (porém facultativo), específico para cobrir o roubo de cargas. A Tabela 1 apresenta mais informações sobre esses seguros.

Tabela 1: Características gerais dos seguros de carga para o Transporte Rodoviário

\begin{tabular}{|c|c|c|}
\hline Seguro de Carga & A quem se destina & Cobertura \\
\hline $\begin{array}{l}\text { Riscos Rodoviários } \\
\text { (RR) } \\
\text { (cobertura básica do } \\
\text { Seguro } \\
\text { Transporte } \\
\text { Terrestre) }\end{array}$ & $\begin{array}{l}\text { Obrigatório para } \\
\text { embarcadores. } \\
\text { Pode ser utilizado } \\
\text { por } \\
\text { transportadores }\end{array}$ & $\begin{array}{l}\text { É um seguro all risks }{ }^{1} \text {. De uma } \\
\text { maneira geral, não cobre apenas } \\
\text { o vício próprio da mercadoria, a } \\
\text { imperícia, imprudência ou } \\
\text { negligência de transportadores e } \\
\text { embarcadores. } \\
\text { Cobre o roubo de cargas quando } \\
\text { constatada a "força maior". }\end{array}$ \\
\hline $\begin{array}{l}\text { Responsabilidade } \\
\text { Civil do } \\
\text { Transportador } \\
\text { Rodoviário de Carga } \\
\text { (RCTR-C) }\end{array}$ & $\begin{array}{l}\text { Obrigatório } \\
\text { exclusivo para } \\
\text { transportadores }\end{array}$ & $\begin{array}{l}\text { Garante aos transportadores faltas } \\
\text { e avarias sofridas pelos bens que } \\
\text { lhes são entregues para o } \\
\text { transporte e pelos quais sejam } \\
\text { responsáveis em consequência, } \\
\text { basicamente de imperícia, } \\
\text { imprudência ou negligência }\end{array}$ \\
\hline $\begin{array}{l}\text { Facultativo de } \\
\text { Responsabilidade } \\
\text { Civil do } \\
\text { Transportador } \\
\text { Rodoviário por } \\
\text { Desaparecimento de } \\
\text { Carga (RCF-DC) }\end{array}$ & $\begin{array}{l}\text { Facultativo } \\
\text { exclusivo para } \\
\text { transportadores }\end{array}$ & $\begin{array}{l}\text { Garante aos transportadores as } \\
\text { perdas ou danos decorridos do } \\
\text { desaparecimento da carga } \\
\text { concomitantemente com o } \\
\text { veículo transportador, em } \\
\text { consequência de furto simples ou } \\
\text { qualificado; roubo; extorsão } \\
\text { simples ou mediante sequestro; e } \\
\text { apropriação indébita. }\end{array}$ \\
\hline
\end{tabular}

\footnotetext{
${ }^{1}$ Espécie de seguro que cobre uma grande variedade de riscos.

Fonte: adaptado de Funenseg (1994) e Manual Transportes (1994).

ADMINISTRAÇÃO DOS SEGUROS DE CARGA
} 
Para a coleta de dados junto às empresas, a técnica de interrogação utilizada foi o questionário, enviado via correio. A elaboração do questionário seguiu as recomendações de Sellitz et al. (1987) e Goode \& Hatt (1968). A amostra de 200 empresas foi obtida através de sorteio, tendo como fonte de informações o banco de dados do Sistema de Informações de Fretes para Cargas Agrícolas ${ }^{4}$ (SIFRECA).

As variáveis utilizadas neste trabalho constam na Tabela 2.

Tabela 2. Descrição das variáveis utilizadas.

\begin{tabular}{ll}
\hline Variável & Sigla \\
\hline Tipo de Carga & TCARGA \\
Tamanho da Frota & TFROTA \\
Especialização na administração dos seguros & ESPSEG \\
Opinião sobre a administração dos seguros & ADMSEG \\
Comportamento do Embarcador para o seguro RR & COMPRR \\
Serviço prestado pelo corretor de seguros & SERVCO \\
\hline
\end{tabular}

A hipótese de que estaria havendo uma "falta de conhecimento" dos transportadores em relação ao serviço de seguro de carga é bastante difícil de ser verificada pela sua natureza subjetiva.

Entende-se por "conhecimento do serviço de seguro" não apenas o conhecimento da sua operacionalização, das cláusulas contratuais e de suas coberturas, mas também a concepção de sua importância para a atividade de transporte, principalmente no relacionamento entre transportadores e embarcadores.

O contrato de seguro de carga geralmente não é um contrato trivial, ou seja, a própria natureza da atividade de transporte exige a manutenção de um contrato aberto, em que os fretes são averbados a cada viagem. Além disso, os prêmios cobrados variam significativamente conforme algumas variáveis, tais como o tipo de carga e as regiões de origem e destino do frete. (Fischer, s.d.). Sendo assim, podem existir contratos complexos, que acabam por exigir uma atenção constante e minuciosa por parte das empresas, situação essa em que deveria haver um indivíduo, ou um grupo deles, exclusivamente alocados para a execução dessa tarefa.

Todavia, a especialização na administração de uma carteira de seguro de carga pode não ser viável em determinados casos, como por exemplo, nas empresas de pequeno porte. Nesses casos, a tarefa pode ser executada por funcionários que também desempenham outras funções dentro da empresa, como por exemplo, os contadores. Ou então - e isso é bastante comum - os próprios proprietários assumem essa função.

Para efeito da análise desses fatos no âmbito desta pesquisa, uma das formas encontradas foi o questionamento sobre essa "dificuldade" encontrada pelos indivíduos em relação à administração da carteira de seguro de carga das empresas. Essa informação é tomada como uma indicação do "conhecimento" desses indivíduos em relação ao assunto.

\footnotetext{
${ }^{4}$ O SIFRECA é um projeto de pesquisa desenvolvido pelo Departamento de Economia, Administração e Sociologia da Escola Superior de Agricultura "Luiz de Queiroz" (USP), que tem como objetivo principal, o levantamento periódico de informações de valores de fretes para cargas agrícolas e agroindustriais no Brasil, para os diferentes modais de transporte.
} 
Obviamente que a relação entre esses dois atributos - "desconhecimento" e "dificuldade" - não é assim tão direta, pois um indivíduo pode não ter dificuldade com determinada tarefa, mas ao mesmo tempo, executá-la de uma forma completamente equivocada. De qualquer maneira, foi a alternativa encontrada para o propósito.

A variável ESPSEG identifica as empresas que apresentam um indivíduo ou um departamento específico que seja responsável pela administração dos seguros, ou seja, identifica aquelas empresas onde espera-se existir uma certa especialização na condução dessa tarefa. De uma maneira geral, 70\% das empresas não dispõem desse serviço especializado.

É importante, porém, que essa informação seja analisada considerando-se o porte das empresas. Os dados agregados por tamanho de frota mostram que há uma relação direta entre a especialização na administração dos seguros de transporte e o tamanho das empresas.

No caso das pequenas, apenas $19 \%$ possuem essa característica. No grupo das empresas de grande porte, esse percentual é de 38\%. É importante mencionar, contudo, que dentre as grandes empresas da amostra, praticamente todas aquelas que operam com mais de 500 veículos, dispõem desse serviço especializado.

Conclui-se assim, que a especialização das tarefas relacionadas à operacionalização dos contratos de seguro de carga pode ser considerada característica de um grupo relativamente pequeno de empresas.

Uma outra variável relacionada aos aspectos administrativos é a $A D M S E G$, que contém informações sobre a impressão dos indivíduos em relação à operacionalização (administração) dos seguros de carga. Tal variável é composta por dois atributos: "dificuldade" e "burocracia". A Tabela 3 ilustra a participação das empresas conforme essa variável.

\section{Tabela 3. Participação das empresas segundo a variável ADMSEG (impressão sobre a} administração dos seguros).

\begin{tabular}{lll}
\hline \multirow{2}{*}{ Burocracia } & $\begin{array}{l}\text { Dificuldade } \\
\text { Pequena } \\
(A D M S E G=\text { fácil })\end{array}$ & $\begin{array}{l}\text { Grande } \\
(A D M S E G=\text { difícil })\end{array}$ \\
\cline { 2 - 3 } Não Burocrática & $34,0 \%$ & $8,5 \%$ \\
Burocrática & $41,0 \%$ & $16,5 \%$ \\
\hline
\end{tabular}

A alternativa mais escolhida pelas empresas foi aquela que diz que a administração dos seguros de transporte é "fácil, porém burocrática". Segundo os dados obtidos, 41\% das empresas se enquadram nessa categoria. Em seguida aparece a alternativa "fácil e não burocrática", com 34\% das empresas.

Considerando-se os atributos separadamente, percebe-se que, mesmo sendo considerada "fácil" em 75\% dos casos - a administração dos seguros é tida como "burocrática" para 57\% das empresas entrevistadas.

É importante ainda verificar a relação entre a $A D M S E G$ e mais outras três variáveis: o tamanho das empresas (TFROTA), a especialização na administração (ESPSEG), e o serviço dos corretores de seguros (SERVCO). 
Em relação ao tamanho das empresas, agregando-as como anteriormente, obtém-se as informações de participação das mesmas em relação à $A D M S E G$. Essas participações estão contidas na Tabela 4.

Tabela 4. Participação das empresas, separadas pelo tamanho de sua frota, segundo a variável ADMSEG (impressão sobre a administração dos seguros).

\begin{tabular}{llll}
\hline$A D M S E G$ & Pequenas & Médias & Grandes \\
\hline Difícil & $26,67 \%$ & $24,68 \%$ & $18,75 \%$ \\
Burocrática & $61,33 \%$ & $58,44 \%$ & $46,88 \%$ \\
\hline
\end{tabular}

Assim, as empresas de menor porte tendem a avaliar como "mais difícil" e "mais burocrática" a administração dos seguros, que as grandes empresas. Essa diferença não parece ser muito significativa entre as pequenas e médias empresas.

Quando comparadas as variações em um mesmo atributo, entre categorias de empresas, percebese que a diferença de impressão em relação à "burocracia" é maior do que a de "dificuldade". Em síntese, isso significaria que as empresas menores "sofrem" relativamente mais com os aspectos burocráticos, que com a dificuldade que têm na execução da tarefa.

Finalmente devem ser consideradas, conjuntamente, as variáveis a ADMSEG e a ESPSEG. Ambas estão intimamente ligadas e devem apresentar alguma relação entre si. A Tabela 5 mostra a participação das empresas conforme os valores assumidos por essas variáveis.

Tabela 5. Participação das empresas segundo as variáveis $A D M S E G$ (impressão sobre a administração dos seguros) e ESPSEG (especialização na administração dos seguros), analisadas de forma conjunta.

\begin{tabular}{lll}
\hline ADMSEG & ESPSEG & \\
& com especialização & sem especialização \\
\hline Difícil & $16,7 \%$ & $28,9 \%$ \\
Burocrática & $58,3 \%$ & $57,0 \%$ \\
\hline
\end{tabular}

A relação que existe entre as variáveis ADMSEG e ESPSEG refere-se ao atributo "dificuldade", onde a não-especialização na administração de seguros é responsável pela existência de um número maior de empresas que enfrenta dificuldade na execução dessa tarefa. De acordo com a Tabela 5, apenas 16,7\% das empresas que possuem uma certa especialização com os seguros manifestaram alguma dificuldade em relação a administração desses serviços, enquanto que - quando essa especialização não existe - esse percentual passa para 28,9\%.

Esse resultado - da relação $A D M S E G$ vs. ESPSEG - ao que tudo indica, ainda confirma o fato que a manutenção e operacionalização de uma apólice de seguro de carga (principalmente averbações) é naturalmente, ou melhor, intrinsecamente, minuciosa, exigente de atenção especial e, consequentemente, reconhecida como "burocrática", independentemente da existência ou não de um serviço especializado para o seu tratamento. 
Além desse aspecto da manutenção das apólices, deve-se considerar os trâmites necessários quando da ocorrência de algum sinistro com determinada carga. Seguidamente os indivíduos lembram os procedimentos burocráticos necessários para a obtenção da indenização securitária.

O que chama a atenção, contudo, é o fato de que a diferença em relação à "burocracia", mesmo que não tenha sido verificada quando da consideração da existência ou não de especialização, foi encontrada entre empresas de portes diferentes.

Uma última variável a ser considerada na análise da impressão dos transportadores em relação à administração dos seguros é o serviço prestado pelos seus respectivos corretores de seguros (SERVCO).

$\mathrm{O}$ corretor de seguros, no caso do transporte rodoviário de carga, apresenta uma função ainda mais evidente que em outros ramos, seja pelas características naturais desse seguro, seja pelo fato de que esse indivíduo também acaba desenvolvendo um papel de orientação às formas de gerência de risco adotada pelos transportadores. Certamente essas duas funções estão intimamente relacionadas, mesmo porque a oferta dos seguros pode estar condicionada à adoção de alguns desses procedimentos. No momento, contudo, o que deve ficar claro é que a existência de um serviço - de qualidade - na corretagem, é fundamental para o desenvolvimento da atividade de transporte de carga.

O contato com algumas empresas estudadas mostrou, também, que são comuns os casos em que os transportadores delegam aos seus corretores, toda a responsabilidade pela operacionalização da sua carteira de seguros de carga, ou seja, tais situações demonstram a confiança - e consequentemente, a importância - do serviço de corretagem de seguros.

Mesmo nos casos em que essa relação não é assim tão estreita, espera-se que uma adequada prestação do serviço de corretagem deva facilitar sensivelmente a administração dos seguros por parte dos transportadores. Em outras palavras - e voltando ao propósito inicial - deve-se verificar o grau de relação entre a "satisfação" dos transportadores em relação aos seus corretores - captada pela variável $S E R V C O$ - e a avaliação desses transportadores em relação à administração dos seguros (ADMSEG).

Primeiramente, a Figura 2 ilustra a participação das empresas conforme a variável SERVCO.

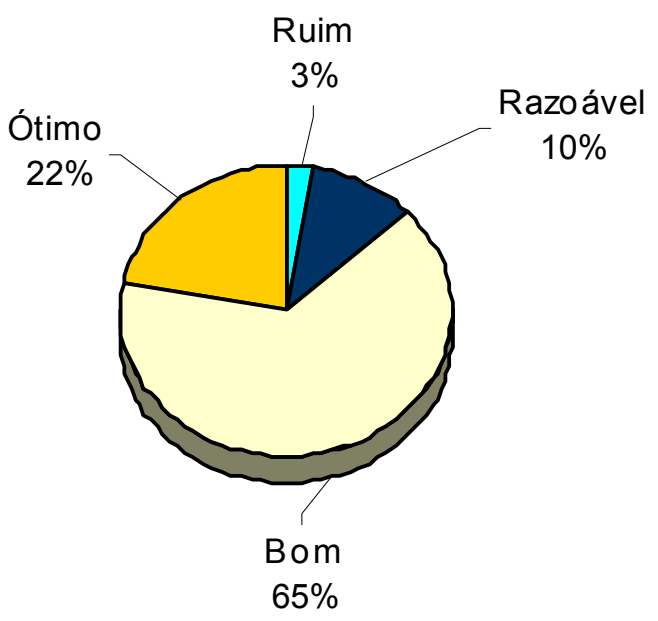

Figura 2. Participação das empresas da amostra segundo a impressão que têm sobre o serviço prestado por seu corretor de seguros (SERVCO). 


\section{GESTÃO DE OPERAÇÕES E LOGÍSTICA - ADMINISTRAÇÃO DE SEGUROS NO TRANSPORTE DE CARGAS} Augusto Hauber Gameiro - José Vicente Caixeta Fiho

As informações obtidas mostram que, de uma maneira geral, as empresas transportadoras parecem estar satisfeitas com o serviço prestado por seus respectivos corretores. A explicação mais provável para esse fato deve ser a importância que esses corretores representam para a atividade, o que estaria se refletindo em um reconhecimento por parte dos transportadores.

Portanto, mesmo com a existência de problemas de oferta ${ }^{5}$ de RCF-DC (o "seguro de roubo") e, com a imposição de uma série de exigências por parte das corretoras/seguradoras para a oferta desse seguro - o que estaria criando um sentimento de conflito, ou até mesmo "antipatia" - os transportadores apresentam uma impressão positiva do serviço prestado por essas empresas.

Ao se considerar a relação entre as variáveis SERVCO e ADMSEG, percebe-se que os transportadores que consideram que o serviço de seus corretores seja de melhor qualidade apresentam uma impressão de que a administração dos seguros é "mais fácil" e "menos burocrática", de acordo com os dados obtidos, sendo que o efeito dessa especialização é maior sobre o atributo "burocracia" que sobre o atributo "dificuldade".

Essas comparações entre variáveis permitem a indicação de que tanto a especialização "dentro" da empresa de transporte (ESPSEG) , como a especialização do serviço dos corretores de seguros (SERVCO), tendem a apresentar um efeito positivo no desempenho das mesmas no que se refere ao gerenciamento dos assuntos ligados aos seguros de carga.

$\mathrm{Na}$ realidade - e isso deve ficar claro - esses dois atributos podem acabar se confundindo um com o outro, pois sua avaliação por parte dos entrevistados é bastante subjetiva, dependendo muito do que os mesmos consideram como algo difícil e burocrático. Entretanto, visto que os dados espelharam um resultado bastante condizente com o esperado, essa distinção parece ter sido assimilada pelos respondentes.

Uma outra análise relevante, relacionada ao conhecimento dos transportadores, é aquela referente ao comportamento dos embarcadores em relação ao seguro $\mathrm{RR}^{6}$ (o "seguro do embarcador").

O seguro RR sempre deveria ser utilizado pelos embarcadores no transporte de suas mercadorias, independentemente de serem transportados pelos próprios embarcadores ou por transportadores terceiros. Todavia, observa-se que isso não ocorre na maioria das situações. Quando questionados os transportadores a respeito desse fato, suas respostas ratificaram tal idéia.

Neste momento, é necessário um comentário fundamental. Como foi dito, o seguro RR não é exclusivo dos embarcadores, podendo ser utilizado por transportadores. Além disso, é um seguro que apresenta maior número de coberturas que os seguros de responsabilidade civil sendo, portanto, um seguro all risks.

Consequentemente, os embarcadores podem exigi-lo dos transportadores e, em alguns desses casos, em nome dos próprios embarcadores. Isso ocorre, segundo os dados da pesquisa com $8 \%$ das transportadoras.

\footnotetext{
${ }^{5} \mathrm{O}$ elevado risco de ocorrência de roubo de cargas implica em uma forte seleção das empresas transportadoras pelos corretores/seguradores para a oferta do seguro de roubo (RCF-DC). Para maiores detalhes, consultar Gameiro, 1999.

${ }^{6}$ Ressalta-se que "RR" é apenas a cobertura básica do Seguro de Transporte Nacional Terrestre para o setor rodoviário. Utiliza-se a expressão simplificada "Seguro RR" no sentido de facilitar a comunicação.
} 
As situações mais comuns, todavia, são aquelas em que os embarcadores não exigem e nem fazem o RR: 50\% das transportadoras responderam nesse sentido.

Finalmente, 32\% dos entrevistados simplesmente não souberam responder a questão, mostrando, por consequência, um grande desconhecimento em relação ao comportamento do embarcador no que concerne à utilização do seguro RR. Esse fato fortalece a idéia de que a hipótese aqui analisada não pode ser refutada.

\section{CONCLUSÕES}

A especialização na administração de seguros de carga nas empresas de transporte é característica de um pequeno número de empresas. Isso acaba sendo prejudicial ao setor no que se refere ao gerenciamento do risco, principalmente o de roubo, pois as empresas podem não estar utilizando as coberturas adequadas para cada situação, ou nem mesmo cientes da importância do seguro no contexto atual.

Essa "falta de especialização", como era de se esperar, é mais evidente em empresas de menor porte, as quais afirmam terem muita dificuldade na administração dos seguros de carga. Essas empresas, consequentemente, podem se deparar com problemas de oferta ao demandarem seguro para o roubo de cargas, por não apresentarem uma política adequada de gerência de risco aos olhos dos corretores/seguradores.

Uma recomendação nesse sentido seria a criação de produtos de seguros mais adequados (simplificados) às empresas menores. Já há, no mercado, algumas iniciativas ainda bastante modestas nesse sentido. A legislação e regulamentação securitária ainda é bastante rígida no País, o que dificulta medidas nesse sentido.

Outra conclusão do trabalho é a de que a figura do corretor de seguros (intermediário entre o segurador e o segurado) apresenta importante função no setor de transporte, confundindo-se, muitas vezes, com um assessor no que se refere à gerência de risco por parte das empresas. Isso pode ser verificado pelo reconhecimento das empresas ao serviço prestado por esses corretores.

Além disso, constatou-se que as empresas transportadoras que dispunham de um bom serviço de corretagem de seguros, avaliam como mais fácil e menos burocrática a tarefa de administração dos seguros.

A avaliação dos transportadores em relação ao comportamento dos embarcadores também apresentou resultado interessante no sentido de mostrar que estaria havendo uma certa falta de comunicação entre as duas partes.

Segundo as empresas transportadoras, a grande maioria dos embarcadores não está utilizando o seguro que lhes é obrigatório, ficando, consequentemente, uma maior responsabilidade sobre os transportadores.

O roubo de cargas, quando comprovado ter sido um caso do força maior, não mais é caracterizado como responsabilidade do transportador. Nesses casos, o seguro do embarcador - o RR - é que deve ressarci-lo das perdas ocasionadas. Por tratar de um seguro pouco utilizado, há uma grande pressão sobre os transportadores para que sejam responsabilizados pelo ocorrido. Por outro lado, em se 
confirmando a responsabilidade do transportador sobre a ocorrência do evento, o seguro RCF-DC é que deve ser acionado.

Além dessa omissão dos embarcadores, a maioria das empresas transportadoras afirmaram que desconhecem o fato de que as cargas que lhes estão sendo entregues para o transporte, estejam cobertas pelo seguro dos embarcadores. Isso, de certa forma, ilustra a "falta de comunicação" entre embarcadores e transportadores, o que deveria ser revisto pelos mesmos diante dos iminentes riscos da atividade.

A ocorrência de um evento de roubo de carga acaba gerando grandes conflitos entre embarcadores e transportadores. Visando evitar problemas nesse sentido, cada uma das partes deveria utilizar o tipo de seguro mais recomendável. Além do que, esses conflitos são extremamente desgastantes, raramente mantendo a boa a relação comercial até então existente entre as partes.

\section{Artigo recebido em 19/04/1999. Aprovado em 07/11/2001}

\section{BIBLIOGRAFIA}

CASTRO, N. de Estrutura e desempenho do setor de transporte rodoviário de carga Pesquisa e Planejamento Econômico, v.18, n.1, p.55-82, abr. 1988.

FISCHER, H. El Seguro de Transportes de Mercancias. Zurich: Compañia Suiza de Reaseguros, s.d. $204 p$.

FUNENSEG, Fundação Escola Nacional de Seguros. Seguros de Transportes Nacionais. Superintendência de Ensino, Asses. Téc. CARVALHO, M.A.G., Rev. e Atual. RIO-MAYOR, A.J.G. Rio de Janeiro: FUNENSEG, 1994. 253p.

GAMEIRO, A.H. A Demanda por Seguro e o Roubo de Cargas no Transporte Rodoviário Brasileiro. Piracicaba, 1999. 146p. Dissertação (Mestrado) - Escola Superior de Agricultura "Luiz de Queiroz", Universidade de São Paulo.

GOODE, W.J.; HATT, P.K. Métodos em Pesquisa Social. 2.ed. Trad. C.M.Bori São Paulo: Companhia Editora Nacional, 1968. 488p.

INFORME SIFRECA. Piracicaba: ESALQ-USP, maio 1997.

Manual Transportes São Paulo: Editora Manuais Técnicos de Seguros, 1994. 874p.

MEHR, R. I., HEDGES, B. A. Risk Management: Concepts and Aplications Homewood: Irwin, 1963. Cap.1.

MOONEY, J.E. The Perfect Crime for the 1990's: Cargo Theft Risk Management, v.41, p.59-61, Nov. 1994.

SELlTIZ, WRIGHTSMAN, COOK. Métodos de Pesquisa nas Relações Sociais. 4.ed. Trad. M.M.H. d'Oliveira e M.M. del Rey; Coord. J.R. Malufe e B.A. Gatti São Paulo: EPU, 1987. 133p. 
SINDICATO DAS EMPRESAS DE TRANSPORTE DE CARGAS DE SÃO PAULO E REGIÃO (SETCESP) O Roubo de Cargas - Avaliação e Perspectivas. /Apresentado a 1. reunião do Conselho Nacional das Entidades Representativas do Transporte Rodoviário de Cargas (CONET), Canela RS, $1997 /$

UELZE, R. Transporte \& Frotas São Paulo: Pioneira, 1977. 375p. 